\title{
Tomography of core-mantle boundary and lowermost mantle coupled by geodynamics: joint models of shear and compressional velocity
}

\author{
Gaia Soldati ${ }^{1,}$, Lapo Boschi $^{2,3}$, Steve Della Mora $^{4}$, Alessandro M. Forte $^{5}$ \\ ${ }^{1}$ Istituto Nazionale di Geofisica e Vulcanologia, Rome, Italy \\ ${ }^{2}$ Sorbonne Universités, Institut des Sciences de la Terre Paris (iSTeP), Paris, France \\ ${ }^{3}$ CNRS, Institut des Sciences de la Terre Paris (iSTeP), Paris, France \\ ${ }^{4}$ Institute of Geophysics, Department of Earth Sciences, ETH Zürich, Switzerland \\ ${ }^{5}$ GEOTOP, Université du Québec à Montréal, Canada
}

\section{Article history}

Received June 12, 2014; accepted November 28, 2014.

Subject classification: Geological and geophysical evidences of deep processes, Mantle and Core dynamics, Tomography and anisotropy, Inverse methods.

\begin{abstract}
We conduct joint tomographic inversions of $P$ and $S$ travel time observations to obtain models of $\delta v_{P}$ and $\delta v_{S}$ in the entire mantle. We adopt a recently published method which takes into account the geodynamic coupling between mantle heterogeneity and core-mantle boundary (CMB) topography by viscous flow, where sensitivity of the seismic travel times to the $C M B$ is accounted for implicitly in the inversion (i.e. the CMB topography is not explicitly inverted for). The seismic maps of the Earth's mantle and CMB topography that we derive can explain the inverted seismic data while being physically consistent with each other. The approach involved scaling P-wave velocity (more sensitive to the $C M B$ ) to density anomalies, in the assumption that mantle heterogeneity has a purely thermal origin, so that velocity and density heterogeneity are proportional to one another. On the other hand, it has sometimes been suggested that $S$-wave velocity might be more directly sensitive to temperature, while P heterogeneity is more strongly influenced by chemical composition. In the present study, we use only $S$-, and not P-velocity, to estimate density heterogeneity through linear scaling, and hence the sensitivity of core-reflected $P$ phases to mantle structure. Regardless of whether density is more closely related to $P$ - or $S$-velocity, we think it is worthwhile to explore both scaling approaches in our efforts to explain seismic data. The similarity of the results presented in this study to those obtained by scaling P-velocity to density suggests that compositional anomaly has a limited impact on viscous flow in the deep mantle.
\end{abstract}

\section{Introduction}

Our understanding of the dynamics of Earth's mantle is largely based on a precise imaging of its velocity structure. Combining tomographic inversion with geodynamic modelling, and using seismically- inferred density variations as a proxy for $\mathrm{CMB}$ topography, Soldati et al. (2012) obtained mantle $v_{P}$ models which are physically sound (their geodynamic coupling to $\mathrm{CMB}$ topography is accounted for) and which fit the seismic data (ISC data sets of $P$-wave arrivals) at least as well as models obtained from seismic data alone. Their method requires that a tomography model of mantle seismic velocity be interpreted in terms of equivalent density anomalies via a constant or radially varying scaling factor. However, density and velocity heterogeneity are proportional to one another only if mantle heterogeneity is of purely thermal origin, and no compositional heterogeneity is present. This is certainly not strictly true, and it is still questioned to what extent it is a valid approximation of the real Earth (e.g. Karato, 2003; Deschamps and Trampert, 2003; Trampert et al., 2004; Della Mora et al., 2011), at least for the uppermost and lowermost regions of the mantle.

Shear and compressional velocity are in principle sensitive to both composition and temperature, but we do not know a priori the relative importance of the two effects. While Soldati et al. (2012) assume a linear relationship between temperature $T$ and $v_{P}$ anomalies, we investigate here the other end-member model of density/velocity scaling, constraining $T$ and $v_{S}$. Our goal is simply to try and explain seismic data with a broader range of seismic/geodynamic models, and we do not claim at this point that $T$ and density are more closely related to $v_{S}$ than to $v_{P}$ (or vice-versa).

We present here a new method to conduct joint 
inversions of $P$ - and $S$-waves, which are no longer coupled via any assumption on their scaling; they are now indirectly coupled through the CMB, which is computed by integration of the $\delta v_{S}$ heterogeneity structure weighted by the geodynamic sensitivity kernels (Forte and Peltier, 1991). This is different from previous joint $P$-S tomographic models (e.g., $S u$ and Dziewonski, 1997; Vasco and Johnson, 1998; Kennett et al., 1998; Saltzer et al., 2001; Kennett and Gorbatov, 2004; Houser et al., 2008), in that the inversion is now also constrained by the expected physical coupling between mantle and CMB, and no a-priori value for $\delta v_{S}$-to- $\delta v_{P}$ scaling is prescribed. Furthermore, only $v_{S}$, and not $v_{P}$, is attached to density structure, while $v_{P}$ heterogeneities are completely free parameters. The assumption that $\delta v_{S}$ (rather than $\delta v_{P}$ ) be proportional to density anomaly is motivated by the observation of, e.g., Boschi et al. $(2007,2008)$ that the distribution of deep plume roots correlates much better with lowermost-mantle $\delta v_{S}$ than $\delta v_{P}$. While density/velocity scaling in the mantle remains an open question, this could be an indication that $\delta v_{S}$ is more sensitive than $\delta v_{P}$ to thermal variation, while $\delta v_{P}$ is more strongly affected by chemical heterogeneity.

The mantle and CMB models we find following this new hybrid approach after scaling $v_{S}$ velocity to density are almost coincident with the ones obtained by Soldati et al. (2012) scaling $v_{P}$ to density; this is a strong indication that the potential presence of compositional heterogeneity in the lowermost mantle, while it may have important local effects, does not heavily affect the viscous convective flow (Simmons et al., 2009).

\section{Method}

We assume a linear relationship between travel time data and seismic velocities (Boschi and Dziewonski, 2000), and use the LSQR method (Paige and Saunders, 1982) to iteratively invert for $\delta v_{P}$ and $\delta v_{S}$ the following system of equations

$$
\begin{gathered}
\delta t_{P}=\int_{\text {path }} \frac{\delta v P(r(s), \Theta(s), \Phi(s))}{v_{P}^{2}(r)} d s \\
\delta t_{P}=-\int_{\text {path }} \frac{\delta v_{P}(r(s), \Theta(s), \Phi(s))}{v_{P}^{2}(r)} d s \\
\delta t_{P c P}=-\int_{\text {path }} \frac{\delta v_{P}(r(s), \Theta(s), \Phi(s))}{v_{P}^{2}(r)} d s+K_{P c P} \frac{\delta c\left(\Theta_{b}, \Phi_{b}\right)}{c}(3)
\end{gathered}
$$

where $r=r(s), \theta=\theta(s), \phi=\phi(s)$ is the ray-path equation, $\left(\theta_{b}, \phi_{b}\right)$ are the coordinates at which the $P c P$ raypath is reflected off the $\mathrm{CMB}$, and $K_{P c P}$ the sensitivity of $\delta t$ to CMB undulations (defined e.g. by Dziewonski and Gilbert (1976)).

The CMB topography $\delta c$ is the other unknown function to be determined; Forte and Peltier (1991) show that its spherical harmonic coefficients $\delta c_{l m}$ coincide with the harmonic coefficients $\delta \rho_{l m}$ of density perturbation modulated by the viscositydependent CMB sensitivity kernels $B_{l}(r)$

$$
\delta c_{l m}=\frac{1}{\Delta \rho_{c m b}} \int_{c}^{a} B_{l}(r) \delta \rho_{l m}(r) d r
$$

with $c$ and $a$ denoting the reference, mean radii of the $\mathrm{CMB}$ and Earth's surface, respectively, and $\Delta \rho_{c m b}$ the density jump across the CMB according to PREM (Dziewonski and Anderson, 1981). The sensitivity kernels $B_{l}$ are computed adopting the radial viscosity profile selected by Mitrovica and Forte (1997) on the basis of the fit to geoid and post-glacial rebound data, neglecting the effect of lateral viscosity variations (Moucha et al., 2007).

Replacing $\delta c$ in Equation (3) with its harmonic expansion 4 , and expressing $\delta v_{P} / v_{P}$ and $\delta v_{S} / v_{S}$ as a linear combination of voxels (Soldati et al., 2012), the system of equations above may be summarized in the compact formula

$$
\left(\begin{array}{c}
\delta t_{S} \\
\delta t_{P} \\
\delta t_{P C P}
\end{array}\right)=\left(\begin{array}{cc}
0 & A_{\text {mantle }}^{S} \\
A_{\text {mantle }}^{P} & 0 \\
A_{\text {mantle }}^{P C P} & A_{C M B}^{P C P} K_{P C P}
\end{array}\right) \cdot\left(\begin{array}{l}
\delta v_{P} \\
\delta v_{S}
\end{array}\right),
$$

where the submatrices $A_{\text {mantle }}^{p}$ and $A_{C M B}^{p}$ represent the sensitivity of the seismic phase $p(S, P, P c P)$ to mantle and $\mathrm{CMB}$ structure, respectively. Taking into account the mechanical relationship between deep mantle heterogeneity and $\mathrm{CMB}$ deflections allows the equations for $\delta v_{P}$ and $\delta v_{S}$ to be coupled via the sensitivity kernels $K_{P c P}$, and the resulting velocity models to be physically consistent with each other and with the CMB topography, obtained from Equation (4) after scaling $\delta v_{S}$ to $\delta \rho$. Relative density anomalies are indeed assumed to be proportional to shear-velocity ones through the constant factor $\delta \ln \rho / \delta \ln v_{S}=0.27$ (average of the profile proposed by Karato (1993) on the basis of mineralogical experiments). This simplistic assumption has been proven to be a good approximation of the mineral properties of the mantle through a series of experiments using the depthdependent scaling factors employed by Simmons et al. (2009) and shown in their Figure 3.

We employ a database of $\sim 630,000$ summary rays travel times of $P$ waves and $\sim 63,000$ of $P c P$ waves extracted from the International Seismological Centre (ISC) bulletin, as corrected by Antolik et al. (2001), plus 


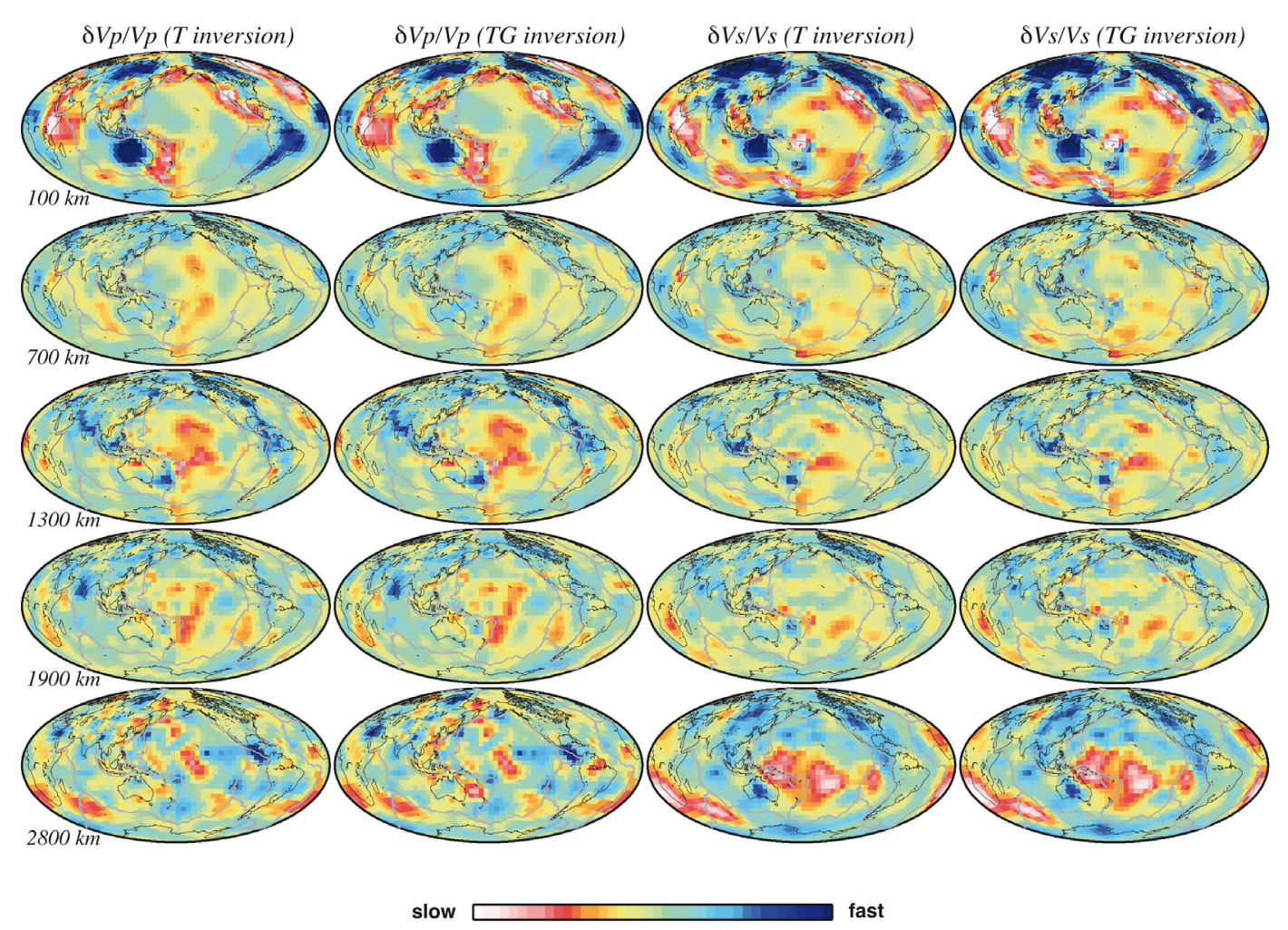

Figure 1. Maps of relative $v_{P}$ and $v_{S}$ velocity variations (\%) at (top to bottom) five different depths in the mantle, obtained from the entire $P, P c P, S$ data set. The maps are derived by jointly inverting ISC and Houser et al. (2008) data with a purely tomographic approach $T$ (columns $1,3$ ), and with a tomographic-geodynamic approach $T G$ (columns 2,4$)$. The scale for the $\delta v_{P}$ maps is $\pm 2 \%$ at $100 \mathrm{~km}$ depth and $\pm 1 \%$ elsewhere, that for the $\delta v_{S}$ maps is $\pm 4 \%$ at $100 \mathrm{~km}$ depth and $\pm 3 \%$ elsewhere; blue regions denote higher than average velocity, and red regions denote lower than average velocity.

the $\sim 170,000 S$ waves arrivals computed by Houser et al. (2008) via a cross correlation technique. This is a preliminary data set that we use in a first exploration of our new methodology; a more complete data set of core-related phases will be assembled in the future. With respect to our previous study (Soldati et al., 2012), we neglect here the data set of PKP travel times because they are also sensitive to possible outer core structure. The resolving power of the data employed has been discussed in detail in Soldati et al. (2012) (see their Figures

\begin{tabular}{cccc}
\hline $\begin{array}{c}\text { Mantle/ } \\
\text { CMB model }\end{array}$ & $\boldsymbol{S}(\mathbf{\%})$ & $\boldsymbol{P ( \% )}$ & $\boldsymbol{P c P ( \% )}$ \\
\hline$\delta v_{P}(T)$ & $/$ & 25.4 & 10.2 \\
$\delta v_{S}(T)$ & 60.3 & $/$ & \\
& & & \\
$\delta v_{P}, \delta v_{S}(T G)$ & 61.3 & 25.3 & 10.5 \\
\hline
\end{tabular}

Table 1. Variance reductions of different databases (columns) achieved by models $T$ and $T G$, as indicated. Note that model $T$ consists of the results of two entirely decoupled inversions (first and second row), as in Della Mora et al. (2011), while model TG (third row) is an individual, joint model including both $v_{S}$ and $v_{P}$ anomalies.
4 and 5). The combination of the $P$ - and $S$-wave data sets provides a better coverage throughout most of the mantle and allows us to get a significant increase in information. The velocity models are parametrized in terms of 15 layers (200 km-thick) of 1656 equal area voxels each (plus 1656 pixels to describe the CMB topography), measuring $5^{\circ} \times 5^{\circ}$ at the equator.

Multi-parameter inversions may be strongly influenced by factors like the weighting associated with different data or the misfit functions. Following Boschi and Dziewonski (1999); Soldati et al. (2012), we assign to each travel time a weight (exponential function) depending on its deviation from PREM predictions. We select different values for the cutoff of our subsets of data, depending on their standard deviation, while we assume the same relative weight for each data set, despite the difference in the number of phase arrivals. The LSQR linearized inversion is regularized via radial and lateral roughness damping only (e.g. Boschi and Dziewonski, 1999).

\section{Results}

3.1. Mantle velocity and CMB topography models We first compute $\delta v_{P}$ and $\delta v_{S}$ models of the Earth's mantle and the corresponding CMB topography map via classic tomographic $(T)$ inversions of the entire 
data set. In this case the inverse problem 5 is totally decoupled (the geodynamic part of the matrix being null) and therefore equivalent to separate inversions of the $S, P, P c P$ data sets. The solution model $\delta v_{P}$, shown in Figure 1 (column 1), is consistent with previously published ones (e.g. Boschi and Dziewonski, 1999, 2000; Tkalĉić and Romanowicz, 2002; Tkalciić et al., 2002; Soldati et al., 2003; Young et al., 2013). The same holds true for the model of mantle $\delta v_{S}$ anomalies (column 3), in close agreement with the results found by Kennett et al. (1998); Ritsema et al. (1999); Houser et al. (2008); Soldati et al. (2012); Auer et al. (2014). Compared to the $\delta v_{P}$ solutions, $\delta v_{S}$ anomalies are considerably larger and the slow structures beneath Southern Africa and Pacific Ocean emerge much more clearly; this suggests a stronger sensitivity of $v_{S}$ to thermal anomalies.

We also show in Figure 1 the results of inverting the entire data set with the tomographic-geodynamic (TG) approach described in Section 2, assuming the radial viscosity profile by Mitrovica and Forte (1997). The $\delta v_{P}$ and $\delta v_{S}$ anomaly maps so obtained (column 2, 4 , respectively) are almost coincident with the corresponding $T$ models, derived without considering the mantle-CMB coupling.

Table 1 shows the variance reductions of inverted data associated with our models. As a general rule, inverting different data sets jointly results in lower variance reduction with respect to inversions of a single data set. The value of $P c P$ variance reductions are only slightly lower than those found by Soldati et al. (2012) (Figure 10), while the variance reduction of $P$ data is more significantly reduced. The same is true for $S$-data variance reduction with respect to the recent model of Auer et al. (2014), who inverted similar data; but notice that Auer et al.'s (2014) model included a much higher number of free parameters, since it allows for radial anisotropy and is parameterized in terms of an adaptive-resolution grid often much denser than ours.

The TG model of $\delta v_{P}$ achieves similar or higher variance reduction to the different subsets of data than that associated with the purely tomographic $T$ one, and similarly the TG model of $\delta v_{S}$ fits the $S$ data better than the corresponding $T$ model. This is a nontrivial result, since the number of free parameters is reduced in the $T G$ inversion (e.g. Soldati et al., 2012).

That a model achieves a higher variance reduction with a lower number of free parameters is an indication that the added regularization provided by geodynamic constraints helps the solution to converge to a better model. The $\delta v_{p}$ model of Figure 1 is in agreement with those found by Soldati et al. (2012) using the same approach to invert solely the ISC $P$-wave data set, and scaling $\delta v_{P}$ (instead of $\delta v_{S}$ ) to $\delta \rho$ heterogeneity.

We show in Figure 2a our model of CMB topography, obtained directly from the $T$ inversion, and in $2 \mathrm{~b}$ the model we obtained stepwise from the $T G$ inversion and integration of $\delta v_{S}$ as described by Soldati et al. (2012). Both models are dominated by harmonic degree 2 , corresponding to systematically negative $\mathrm{CMB}$ topography under the circumpacific ring. This is generally consistent with the observations of, e.g., Morelli and Dziewonski (1987); Boschi and Dziewonski (1999); Rodgers and Wahr (1993); Forte et al. (1995); Soldati et al. $(2003,2013)$. With respect to the T model, the $T G$ one is characterized by stronger CMB

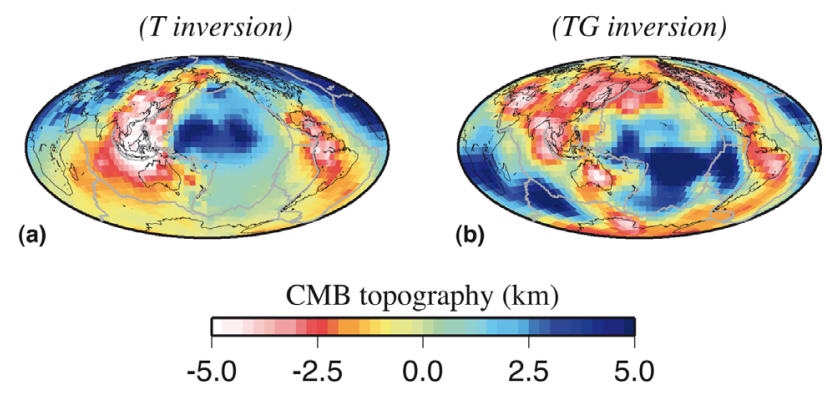

Figure 2. Maps of CMB topography $(\mathrm{km})$ obtained from the entire $P, P c P, S$ data set. The maps are obtained by jointly inverting ISC and Houser et al. (2008) data with a purely tomographic approach $T$ (a), and with a tomographic-geodynamic approach $T G(b)$, integrating the mantle shear velocity anomaly modulated by the sensitivity kernels as in Equation (4). The TG approach assumes a laterally constant scaling between seismic velocity $\left(v_{S}\right.$ here) and density.

depression at very high and low latitudes, and an overall smaller amplitude (with a depth- to-valley amplitude of $13.3 \mathrm{~km}$, vs. a value of $14.6 \mathrm{~km}$ for the $T$ model).

\subsection{Comparison of $v_{P}$ and $v_{S}$ models}

We show in Figure 3a the correlation between shear and compressional velocity anomalies and that between shear and bulk sound velocity $\left(v_{\phi}\right)$ anomalies for $T$ and $T G$ mantle models. We observe a positive correlation between $\delta v_{P}$ and $\delta v_{S}$ throughout the mantle, and a corresponding decorrelation between $\delta v_{\phi}$ and $\delta v_{S}$, with little or no difference between $T$ and $T G$ inversions. All the correlation curves tend to decrease in the midmantle and at $D^{\prime \prime}$ depth, as also found by Della Mora et al. (2011) inverting direct $P$ and $S$ waves with a classic tomographic approach and with finer radial parameterization. The simultaneous drop of correlation $\delta v_{P^{-}} \delta v_{S}$ and anticorrelation $\delta v_{\phi^{-}} \delta v_{S}$ at the base of the mantle has been already observed by several studies (e.g. Su and Dziewonski, 1997; Kennett et al., 1998; Becker and Boschi, 2002; Antolik 


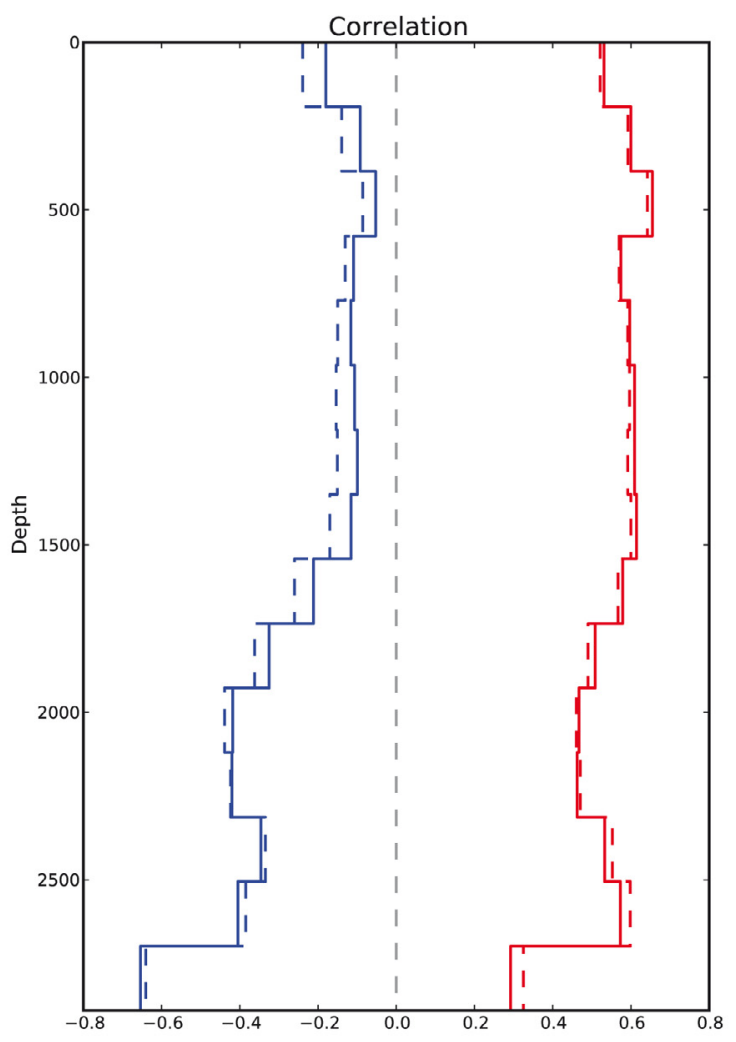

(a)

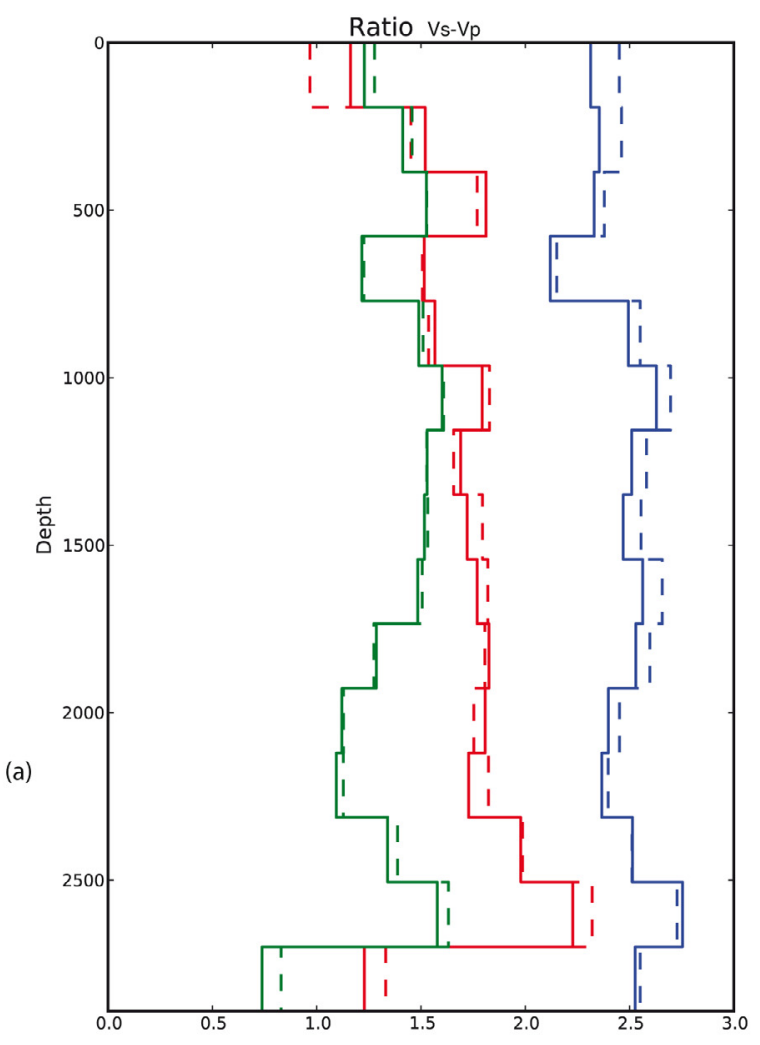

(b)

Figure 3. (a) Radial correlation between $v_{S}$ and $v_{P}$ heterogeneities (red) and between bulk sound velocity and $v_{S}$ heterogeneities (blue), for $T$ (solid) and TG (dashed) mantle models. (b) Ratio between $v_{S}$ and $v_{p}$ heterogeneities as a function of depth (red); same ratio computed via the RMS of both models as a function of depth (blue); same as the latter, but multiplied by the $v_{S}-v_{P}$ correlation coefficients (panel a, red lines) (green). Solid and dashed lines as in panel (a).

et al., 2003; Simmons et al., 2010), and may indicate a non-thermal origin for the velocity heterogeneities (Karato and Karki, 2001; Saltzer et al., 2001; Hirose, 2006).

Another indicator of potential compositional heterogeneity is the ratio $R_{S, P}$ between shear and compressional velocity anomalies, which may be computed following various approaches. Figure $3 \mathrm{~b}$ shows in red the depth dependence of $R_{S, P}$ computed from Equation (1) of Della Mora et al. (2011), while blue and green curves refer to the ratio of the RMS of models $\delta v_{S}$ and $\delta v_{P}$, multiplied/not multiplied by the Pearson's correlation coefficient. This coefficient acts as a weight: wherever the correlation between $\delta v_{P}$ and $\delta v_{S}$ is poor, it is meaningless to assume that they are proportional, and to compute their ratio. Independently from the formula applied, and from the approach used to invert the data $(T / T G), R_{S, P}$ is positive throughout the mantle and increases (of different amounts depending on the formula used to compute it) below $2100 \mathrm{~km}$ depth, with a relative maximum around $2600 \mathrm{~km}$ depth. This trend is consistent with previous studies (Saltzer et al., 2001; Tkalĉić and Romanowicz, 2002; Della Mora et al., 2011), and suggests at least a partial influence of chemical composition on the seismic velocity heterogeneities (Karato, 2003). Again, the importance of compositional effects does not appear to be altered by the inclusion of geodynamic constraints $(T G)$ in the inverse problem.

\section{Discussion}

Using a large data set of $P, P c P$ and $S$ travel time data and the fact that $\mathrm{CMB}$ deflections should be gravitationally related to velocity structure of the deep mantle, we apply the method by Soldati et al. (2012) to joint inversion for both compressional and shear velocity anomalies.

In this approach, CMB topography is not explicitly inverted for, but required to be coupled to mapped mantle heterogeneities according to the viscous flow theory of Forte and Peltier (1991). This is a entirely new approach to joint inversion, in that it does not require any assumption on the scaling between $v_{P}$ and $v_{S}$ anomalies, which are instead only coupled by the CMB. This study represents a significant extension to that of Soldati et al. (2012), in that no scaling was assumed between $v_{P}$ and density anomalies, but rather between $v_{S}$ and density anomalies. Interestingly, despite this important difference in approach, the results of Soldati 
et al. (2012) are confirmed: neither the CMB topography, nor the pattern of mapped low/high velocity are strongly perturbed with respect to theirs. Given the lower sensitivity of $v_{S}$ to compositional heterogeneity, scaling density with $v_{S}$ is equivalent to assume a purely thermal origin of velocity anomalies. Thus, the agreement between mantle models that we obtained by scaling density with $v_{S}$ and $v_{P}$ may interpreted as an indication of the limited influence on mantle flow of compositional heterogeneity. This has been proven even at the African superplume, where, despite the large chemical component of velocity anomalies, the viscous flows appear to be driven by thermal (buoyancy) forces (e.g. Forte and Mitrovica, 2001; Simmons et al., 2009).

The robustness of this result suggests that, while considerable compositional heterogeneity exists in the lowermost mantle, a flow model controlled by temperature/density heterogeneity alone is consistent with the mapped CMB topography and pattern of low/high seismic velocity in the mantle.

Acknowledgements. We thank Hrvoje Tkal i and an anonymous reviewer for helpful reviews of this manuscript.

\section{References}

Antolik, M., G. Ekström, and A. M. Dziewonski (2001), Global event location with full and sparse data sets using three-dimensional models of mantle P-wave velocity, Pure Appl. Geophysics, 158 (1-2), 291-317.

Antolik, M., Y. J. Gu, G. Ekström, and A. M. Dziewonski (2003), J362d28: a new joint model of compressional and shear velocity in the Earth's mantle, Geophysical J. Int., 153 (2), 443-466.

Auer, L., L. Boschi, T. W. Becker, T. Nissen-Meyer, and D. Giardini (2014), Savani: A variable-resolution whole-mantle model of anisotropic shear-velocity variations based on multiple datasets, J. Geophys. Res., 119 (4), 30063034, doi:10.1002/ 2013JB010773.

Becker, T. W., and L. Boschi (2002), A comparison of tomographic and geodynamic mantle models, Geochemistry Geophysics Geosystems, 3; doi:10.12 9/2001GC000,168.

Boschi, L., and A. M. Dziewonski (1999), High and lowresolution images of the Earth's mantle: Implications of different approaches to tomographic modeling, J. Geophys. Res., 104 (B11), 25,567-25,594.

Boschi, L., and A. M. Dziewonski (2000), Whole Earth tomography from delay times of P, PcP, and PKP phases: Lateral heterogeneities in the outer core or radial anisotropy in the mantle?, J. Geophys. Res., 105 (B6), 13,675-13,696.

Boschi, L., T. W. Becker, and B. Steinberger (2007),
Mantle plumes: Dynamic models and seismic images, Geochemistry Geophysics Geosystems, 8, Q10,006, doi:10.10292007GC001733.

Boschi, L., T. W. Becker, and B. Steinberger (2008), On the statistical significance of correlations between synthetic mantle plumes and tomographic models, Phy. Earth. Planet. Int., 167 (3-4), 230-238.

Della Mora, S., L. Boschi, P. J. Tackley, T. Nakagawa, and D. Giardini (2011), Low seismic resolution cannot explain S/P velocity decorrelation in the lower mantle, Geophys. Res. Lett.; doi:10.1029/2011GL 047559 .

Deschamps, F., and J. Trampert (2003), Mantle tomography and its relation to temperature and composition, Phys. Earth Planet. Inter., 140 (4), 277-291.

Dziewonski, A. M., and F. Gilbert (1976), Effect of small, aspherical perturbations on travel times and a re-examination of corrections for ellipticity, Geophys. J. Roy. Astron. Soc., 44 (1), 7-17.

Dziewonski, A. M., and D. L. Anderson (1981), Preliminary reference earth model, Phy. Earth. Planet. Int., 25 (4), 297-356.

Forte, A. M., and W. L. Peltier (1991), Viscous-flow models of global geophysical observables 1: Forward problems, J. Geophys. Res., 96, 20,131-20,159.

Forte, A. M., J. X. Mitrovica, and R. L. Woodward (1995), Seismic-geodynamic determination of the origin of excess ellipticity of the core-mantle boundary, Geophys. Res. Lett., 22 (9), 1013-1016.

Forte, A. M., and J. X. Mitrovica (2001), Deep-mantle high-viscosity flow and thermochemical structure inferred from seismic and geodynamic data, Nature, 410 (6832), 1049-1056.

Hirose, K. (2006), Postperovskite phase transition and its geophysical implications, Rev. Geoph., 44.

Houser, C., G. Masters, P. Shearer, and G. Laske (2008), Shear and compressional velocity models of the mantle from cluster analysis of long-period waveforms, Geophysical J. Int., 174 (1), 195-212.

Karato, S. I., and B. B. Karki (2001), Origin of lateral variation of seismic wave velocities and density in the deep mantle, J. Geophys. Res., 106 (B10), 21,77121,783

Karato, S. I. (2003), The dynamic structure of the deep Earth, Princeton University Press.

Kennett, B. L. N., S. Widiyantoro, and R. D. van der Hilst (1998), Joint seismic tomography for bulk sound and shear wave speed in the Earth's mantle, J. Geophys. Res., 103 (B6), 12,469-12,493.

Kennett, B., and A. Gorbatov (2004), Seismic heterogeneity in the mantle - strong shear wave signature of slabs from joint tomography, Phys. Earth Planet. Inter., 146 (1-2), 87-100; doi:10.1016/j.pepi.2003. 
07.033 .

Mitrovica, J., and A. M. Forte (1997), The radial profile of mantle viscosity: results from the joint inversion of convection and post-glacial rebound observables, J. Geophys. Res., 102, 2751-2769.

Morelli, A., and A. M. Dziewonski (1987), Topography of the core-mantle boundary and lateral homogeneity of the liquid core, Nature, 325, 678-683.

Moucha, R., A. M. Forte, J. X. Mitrovica, and A. Daradich (2007), Lateral variations in mantle rheology: implications for convection related surface observables and inferred viscosity models, Geophys. J. Int., 169 (1), 113-135.

Paige, C., and M. Saunders (1982), LSQR - an algorithm for sparse linear-equations and sparse least-squares, ACM Transactions on Mathematical Software, 8.

Ritsema, J., H. J. V. Heijst, and J. H. Woodhouse (1999), Complex shear wave velocity structure imaged beneath Africa and iceland, Science, 286 (5446), 1925-1928.

Rodgers, A., and J. Wahr (1993), Inference of coremantle boundary topography from ISC PcP and PKP traveltimes, Geophys. J. Int., 115, 991-1011.

Saltzer, R. L., R. D. van der Hilst, and H. Karason (2001), Comparing P and S wave heterogeneity in the mantle, Geophys. Res. Lett., 28 (7), 1335-1338.

Simmons, N. A., A. M. Forte, and S. P. Grand (2009), Joint seismic, geodynamic and mineral physical constraints on three-dimensional mantle heterogeneity: Implications for the relative importance of thermal versus compositional heterogeneity, Geophysical J. Int., 177 (3), 1284-1304.

Simmons, N. A., A. M. Forte, L. Boschi, and S. P. Grand (2010), GyPSuM: A joint tomographic model of mantle density and seismic wave speeds., J. Geophys. Res., 115, B12,310; doi:10.1029/2010JB007,631.

Soldati, G., L. Boschi, and A. Piersanti (2003), Outer core density heterogeneity and the discrepancy between PKP and PcP travel time observations, Geophys. Res. Lett., 30 (4), 1190.

Soldati, G., L. Boschi, and A. Forte (2012), Tomography of core-mantle boundary and lowermost mantle coupled by geodynamics, Geophys. J. Int.; doi:10.1111/j.1365-246X.2012. 05413.x.

Soldati, G., P. Koelemeijer, L. Boschi, and A. Deuss (2013), Constraints on core-mantle boundary topography from normal mode splitting, Geochemistry Geophysics Geosystems, 14 (20); doi:10.1002/ ggge.20115.

Su, W. J., and A. M. Dziewonski (1997), Simultaneous inversion for 3-D variations in shear and bulk velocity in the mantle, Phy. Earth. Planet. Int., 100 (1-4), 135-156.
Tkalciić, H., and B. Romanowicz (2002), Short scale heterogeneity in the lowermost mantle: insights from PcP-P and ScS-S data, Earth and Planetary Science Lettters, 201, 57-68.

Tkalciić, H., B. Romanowicz, and N. Houy (2002), Constraints on $D^{\prime \prime}$ structure using $\operatorname{PKP}(\mathrm{ABDF})$, PKP(BCDF) and PcPP traveltime data from broadband records, Geophys. Int., 148, 599616.

Trampert, J., F. Deschamps, J. Resovsky, and D. Yuen (2004), Probabilistic tomography maps chemical heterogeneities throughout the lower mantle, Science, 306 (5697), 853-856.

Vasco, D. W., and L. Johnson (1998), Whole earth structure estimated from seismic arrival times, J. Geophys. Res., 103, 2633-2671.

Young, M. K., H. Tkalĉić, T. Bodin, and M. Sambridge (2013), Global P wave tomographyof earths lowermost mantle from partition modeling, J. Geophys. Res., 118, 5467-5486; doi:10.1002/jgrb.50391.

\footnotetext{
${ }^{\star}$ Corresponding author: Gaia Soldati, Istituto Nazionale di Geofisica e Vulcanologia, Rome, Italy; email: gaia.soldati@ingv.it.

(C) 2014 by the Istituto Nazionale di Geofisica e Vulcanologia. All rights reserved.
} 